\title{
Is it Disadvantageous to Teach Forces First in Mechanics?
}

\author{
Andrew Pawl \\ Engineering Physics Department, University of Wisconsin-Platteville, Platteville, WI 53818
}

\begin{abstract}
Interactions are the heart of the mechanics course and forces are the fundamental representation of interactions. This suggests that beginning the introductory mechanics course with the topic of forces is a logical option. The majority of commercial textbooks, however, begin the mechanics course by teaching the concept of acceleration from a kinematical perspective before introducing Newton's 2nd Law. This paper presents evidence that college students in calculus-based introductory mechanics perform equally well when the course begins with forces as they do when it begins with kinematics.
\end{abstract}

Keywords: introductory physics, physics education research PACS: 01.40.Fk, 01.40.G-

\section{INTRODUCTION}

The traditional textbooks for calculus-based introductory mechanics teach the concept of acceleration from a kinematical perspective before teaching the mathematics of forces [1]. This order of topics implies that Newton's 2nd Law represents a mathematical definition of "force" as something that is capable of producing acceleration.

It is alternatively possible to present the mathematical description of forces before a kinematical description of acceleration. In this context, Newton's 2nd Law implies a definition of "acceleration" as the result of unbalanced force. Textbooks by authors with ties to physics education research are divided. Some follow a traditional approach (e.g. [2]), while others introduce the concept of force in a qualitative (e.g. [3]) or even quantitative manner (e.g. [4]) before the presentation of kinematics.

The overwhelming preference of traditional textbooks for the kinematics-first approach sends the message that this topical order produces better outcomes. Certain threads of physics education research, on the other hand, indicate that the forces-first approach to the course might be superior (reflected in the split among PER-informed texts). It is therefore of interest to directly compare the outcomes in courses using the two approaches. This paper briefly summarizes the physics education research that implies a forces-first approach holds promise and presents the results of an experiment that indicates the forces first approach is equally as effective as the traditional kinematics-first approach.

\section{MOTIVATION AND BACKGROUND}

One of the earliest findings of physics education research is that conceptual organization helps shift students away from an equation-hunting approach and toward expertise in physics problem solving. The ability to develop a qualitative understanding of a problem before turning to mathematics is the clearest distinction between experts and novices $[5,6]$. Seminal work by Heller and Reif showed that training students to recognize a disciplinespecific conceptual structure can help them develop expertise in problem solving [7]. Their approach was extended by Hestenes and collaborators in the development of Modeling Physics pedagogy [8].

The narrow focus of introductory mechanics is an advantage from the perspective of teaching students the utility of qualitative understanding. Much of the material taught consists of various ways to describe the translational motion of point particles and the rotational motion of rigid bodies. Every problem involving such motions has exactly the same conceptual structure, requiring the answer to three questions:

1. What is the system of interest?

2. What interactions are experienced by the system?

3 . How can the motion of the system and the interactions that affect it be most efficiently described mathematically?

The conceptual organization of this portion of the mechanics course can be summarized in the concept map presented as Fig. 1. The five vertical branches of this map correspond to the five basic motion descriptions introduced in introductory mechanics: velocity, momentum, kinetic energy, angular velocity and angular momentum. Each of these branches specializes to a preferred system type and a relevant class of interactions. Each branch has an associated conservation law that is realized when the relevant interactions cancel.

Pritchard and collaborators have encoded Fig. 1 in a hierarchy of mechanics models (shown as Fig. 2), which is a central component of Modeling Applied to Problem Solving (MAPS) pedagogy [9] and its prescription of a mechanics-specific "System, Interactions, Model" (S.I.M.) problem solving approach. 


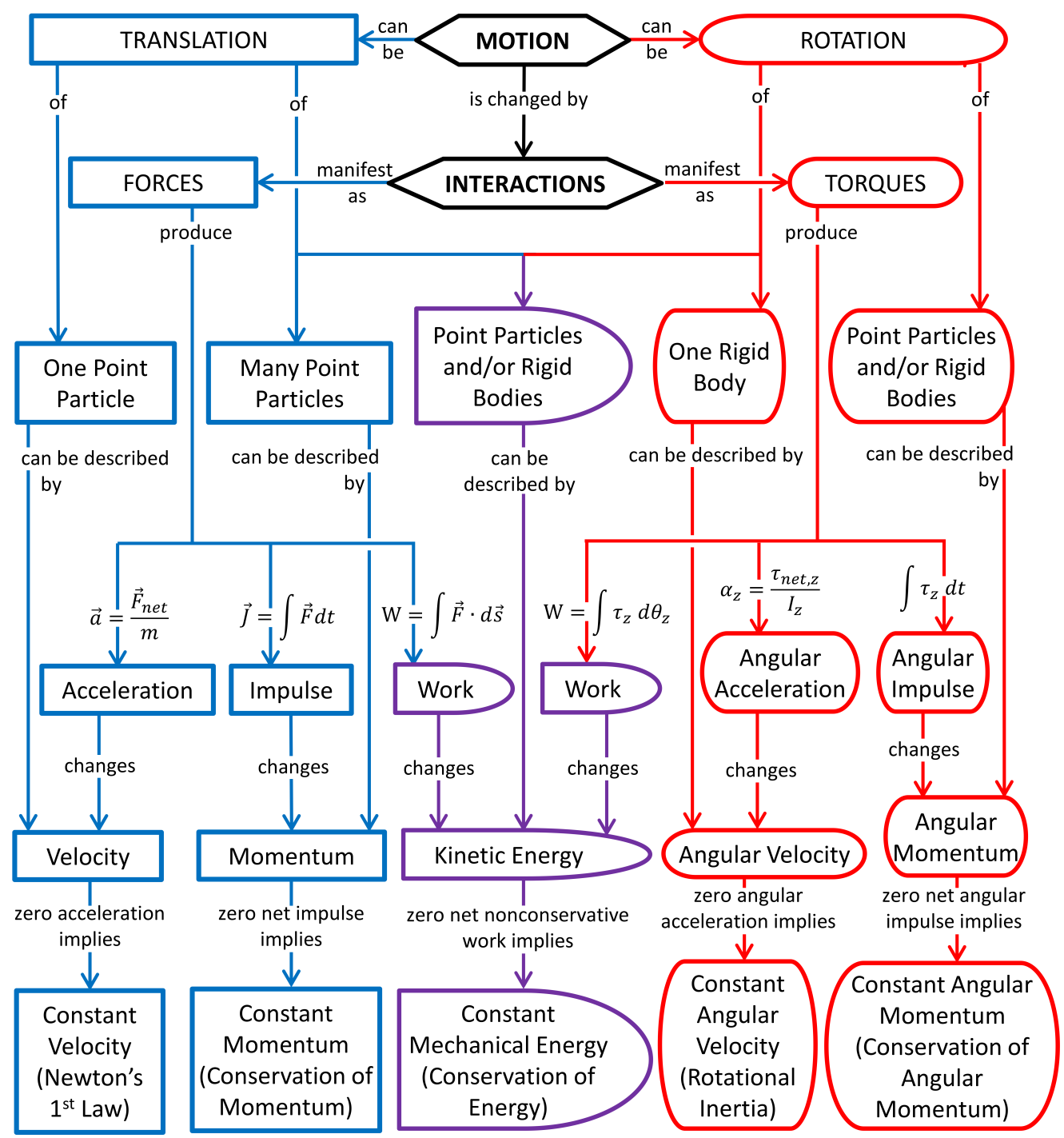

FIGURE 1. Concept map illustrating the structure of mechanics.

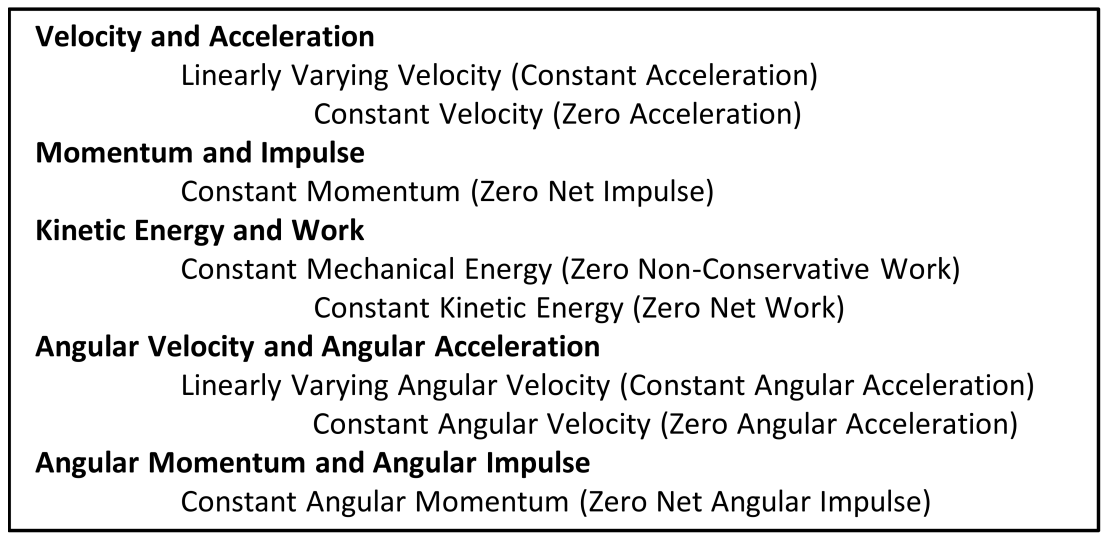

FIGURE 2. Hierarchy of basic models for mechanics. 
MAPS explicitly requires students to identify each component of the S.I.M. framework for each mechanics problem. The System component requires students to explicitly state what objects constitute the system and how they will be approximated. The Interactions component requires that students state what objects interact with the system, identify the type of interaction, and state which of the interactions are relevant to the chosen model. The Model component is a choice from the hierarchy presented in Fig. 2. MAPS pedagogy has been shown to improve the problem solving performance of students who struggled in a traditional mechanics course $[9,10]$.

The success of pedagogies that emphasize the underlying structure of mechanics provides one motivation for teaching forces before acceleration. Force is a generic description of interactions which does not belong to any particular branch of Fig. 1. Acceleration, impulse, or work, by contrast, are branch-specific descriptions of interactions. Teaching forces first allows the teacher to emphasize this point. Teaching acceleration before force and defining force as something that produces an acceleration, on the other hand, gives special status to acceleration as a description of interactions.

\section{AN EXPERIMENT}

In Spring 2013, the author was assigned to teach two sections of the calculus-based mechanics course at the University of Wisconsin-Platteville, a small state school with an engineering program. Each section had an enrollment of over 50 students, primarily engineering majors. The courses were taught in a studio classroom (14 tables of four) with integrated laboratory. Group problems were given once per week. The author had about a decade of experience teaching mechanics by beginning with kinematics and had also taught the forcesfirst approach four times. Teaching two independent sections simultaneously offered the opportunity to test the hypothesis that starting instruction with forces is not a disadvantage to the students. Two different syllabi were constructed for the two sections of mechanics. One was taught kinematics first and the other forces first.

In an effort to control variables, a two-week force sequence and two-week kinematics sequence were designed that could be transposed as closely as possible. The instructional material for weeks 1 and 2 for each of the sections was identical to weeks 3 and 4 of the other (see Table 1), with the exception that the kinematicsfirst section was given two unique kinematics homework problems in week 1 and the forces-first section was given two unique problems involving Newton's 2nd Law in week 2. All material for weeks 5-15 was identical. Thus, over the course of the semester, both sections received the same in-class material and the assigned homework differed by approximately 2 problems out of 70 .
TABLE 1. Order of topics for the first four weeks in each of the two sections that were part of the experiment. Later weeks of both sections were identical.

\begin{tabular}{|c|c|c|}
\hline Week & Kinematics First & Forces First \\
\hline 1 & 1D Kinematics & Equilibrium \\
\hline 2 & 2D Kinematics & Newton's 2nd Law \\
\hline 3 & Equilibrium & 1D Kinematics \\
\hline 4 & Newton's 2nd Law & 2D Kinematics \\
\hline
\end{tabular}

\section{OUTCOMES}

The Force Concept Inventory (FCI) [11] was administered on the first day of instruction in both sections and again on the second-to-last day. Results for both the preand post-instruction administrations as well as the normalized gain [12], defined by:

$$
<g>=\frac{\text { posttest score }- \text { pretest score }}{\text { maximum score }- \text { pretest score }}
$$

are reported in Table 2. No significant difference is observed between the sections in any of these measures.

TABLE 2. Average Force Concept Inventory scores and normalized gains for the two sections involved in the experiment.

\begin{tabular}{|l|l|l|}
\hline FCI Result & $\begin{array}{l}\text { Kinematics First } \\
(\mathrm{N}=39)\end{array}$ & $\begin{array}{l}\text { Forces First } \\
(\mathrm{N}=48)\end{array}$ \\
\hline Pre-Instruction & $11.9 \pm 0.9$ & $13.9 \pm 0.9$ \\
\hline Post-Instruction & $17.8 \pm 0.9$ & $18.8 \pm 0.9$ \\
\hline Normalized Gain & $0.32 \pm 0.06$ & $0.31 \pm 0.07$ \\
\hline
\end{tabular}

Students in both sections were administered identical 3rd and 4th midterm exams and final exams. Average performance and standard deviations for these exams as well as for the overall course grade are shown in Fig. 3. No significant disparities are observed.

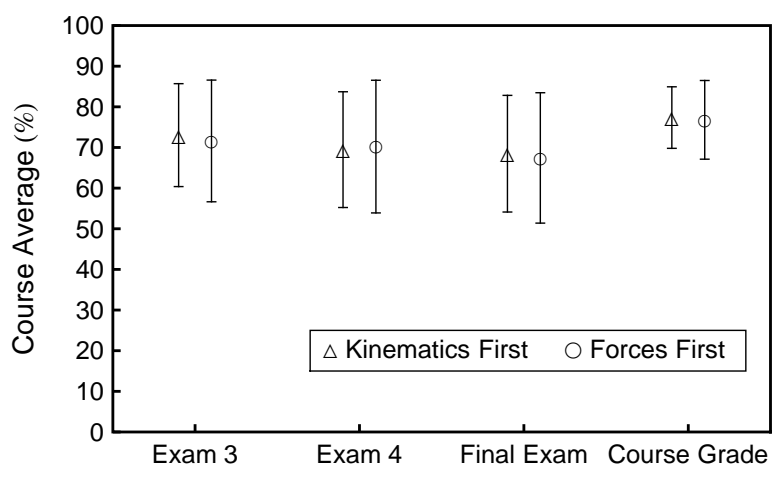

FIGURE 3. Performance on the exams that were identical in content between the two sections. For this plot only, error bars represent the standard deviation of the population rather than the standard deviation of the mean.

The Colorado Learning Attitudes about Science Survey (CLASS) [13] was administered at the beginning and again at the end of the semester. The shifts observed from the pre-instruction to the post-instruction administration in the eight commonly reported categories of statements 
from the CLASS [13] are shown as Fig. 4. The basic pattern of shifts is similar and the shifts observed for seven of the eight categories are not statistically distinguishable. The section which was taught kinematics first does show a shift that is significantly more favorable than the section which was taught forces first in the Problem Solving category of statements, however.

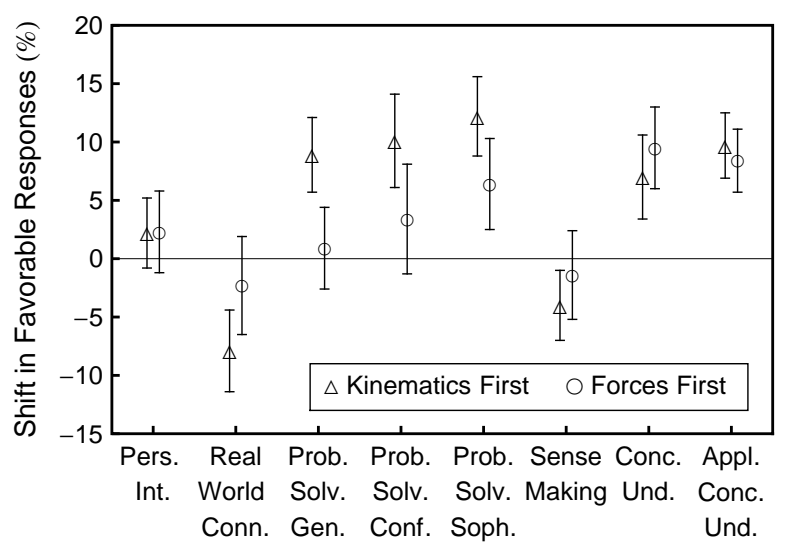

FIGURE 4. Shifts in CLASS responses.

Students were also given the opportunity to evaluate the performance of the instructor using an official form constructed by the department. The results are shown as Fig. 5. No significant disparity is observed.

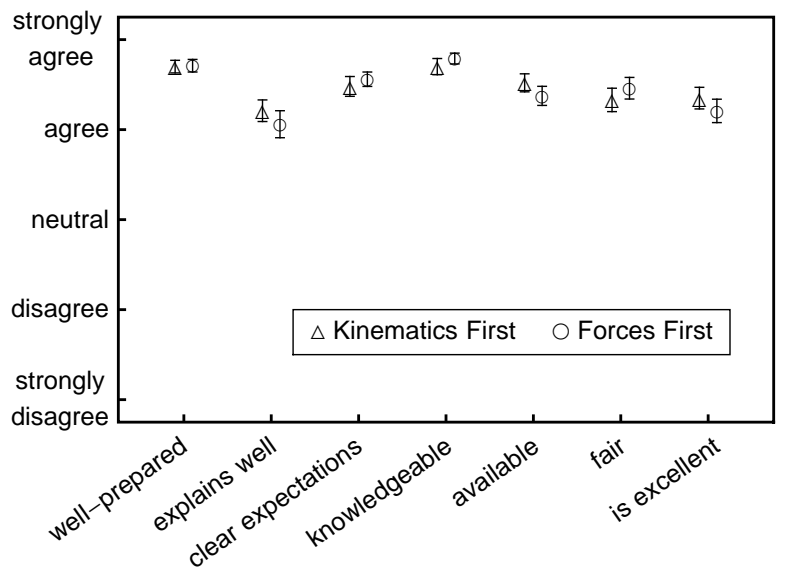

FIGURE 5. Results of instructor evaluations.

\section{CONCLUSIONS AND FUTURE WORK}

The experiment described in this paper shows that when all other variables are controlled, teaching forces first in calculus-based introductory mechanics provides educational outcomes that are nearly indistinguishable from teaching kinematics first. The next step in this research is to add the use of MAPS pedagogy [9, 10], which emphasizes the underlying conceptual structure of mechanics, to the forces-first syllabus. Future work will be to investigate whether making this structure explicit produces learning gains in a typical calculus-based mechanics course.

One caveat to the results reported here is the fact that college-level calculus-based physics enrolls a different population than algebra-based college physics or precollege physics. A survey of students entering the course studied here suggests that a significant majority (approximately $80 \%$ ) have had prior formal exposure to physics and that an even stronger majority can give a basic definition of acceleration prior to instruction (including over $80 \%$ of those who self-report no prior physics instruction). This fact has obvious consequences for the need to introduce kinematics in order to prepare the students for Newton's 2nd Law. It is not clear that the outcomes of the study conducted would be the same in algebra-based physics, and it is particularly dangerous to extend them to a pre-college physics course.

\section{REFERENCES}

1. See, e.g., D. Halliday, R. Resnick and J. Walker, Fundamentals of Physics, 10th ed., (John Wiley \& Sons, New York, 2014); R.A. Serway and J.W. Jewett, Physics for Scientists and Engineers, 9th ed., (Brooks/Cole, Boston, 2014); H.D. Young and R.A. Freedman, Sears and Zemansky's University Physics, 13th ed., (Addison-Wesley, Boston, 2012).

2. R.D. Knight, Physics for Scientists and Engineers: A Strategic Approach, 3rd ed., (Addison-Wesley, Boston, 2013).

3. R.W. Chabay and B.A. Sherwood, Matter \& Interactions, $3 r d$ ed., (John Wiley \& Sons, New York, 2011).

4. T. Moore, Six Ideas that Shaped Physics, 2nd ed., (McGraw-Hill, New York, 2003).

5. J.H. Larkin and F. Reif, Int. J. Sci. Educ. 1, 191-203 (1979).

6. M.T.H. Chi, P. Feltovich and R. Glaser, Cognitive Sci. 5, 121-152 (1981).

7. J.I. Heller and F. Reif, Cognition Instruct. 1, 177-216 (1984).

8. D. Hestenes, Am. J. Phys. 55, 440-454 (1987); D. Hestenes, M. Wells and G. Swackhamer, Am. J. Phys. 63, 606-619 (1995).

9. A. Pawl, A. Barrantes and D. Pritchard, in Proceedings of the 2009 Physics Education Research Conference, M. Sabella, C. Henderson and C. Singh (Eds.), (AIP Conference Proceedings, New York, 2009), pp. 51-54.

10. S. Rayyan, A. Pawl, A. Barrantes, R. Teodorescu and D.E. Pritchard, in Proceedings of the 2010 Physics Education Research Conference, C. Singh, M. Sabella and S. Rebello (Eds.), (AIP Conference Proceedings, New York, 2009), pp. 273-276.

11. D. Hestenes, M. Wells and G. Swackhamer, Phys. Teach. 30, 141-158 (1992).

12. R. Hake, Am. J. Phys. 66, 64-74 (1998).

13. W.K. Adams, K.K. Perkins, N.S. Podolefsky, M. Dubson, N.D. Finkelstein and C.E. Wieman, Phys. Rev. S.T.-Phys. Educ. Res. 2, 010101 (2006). 